\title{
UTILIZAÇÃO DA PLATAFORMA ARDUINO COMO FERRAMENTA DE CONTROLE PARA UM SISTEMA DE NÍVEL DE LÍQUIDOS.
}

\author{
A. H. GIMENES ${ }^{1}$, P. R. A. PEREIRA ${ }^{2}$ \\ ${ }^{1}$ Centro Universitário das Faculdades Associadas de Ensino - UNIFAE, Departamento de \\ Engenharia Química \\ E-mail para contato: antonio-gimenes2010@hotmail.com \\ ${ }^{2}$ Universidade Estadual de Maringá, Departamento de Engenharia Química \\ E-mail para contato: prapereira@hotmail.com
}

\begin{abstract}
RESUMO - No presente artigo é apresentado o processo de implantação de um método automático de controle de nível em um tanque com alimentação constante e controlada, com o intuito de se estudar o seu potencial para a aplicação industrial. Esse método de controle se baseia na utilização de um microcontrolador denominado Arduino, devidamente programado para controlar a alimentação e descarga desse tanque, em função dos níveis máximo e mínimo, por meio de sinais coletados através de dois sensores de nível, instalados internamente na base e no topo do tanque. A implantação de um método de controle automático via Arduino nesse processo em escala piloto, teve por finalidade verificar possíveis contribuições em termos de viabilidade econômica, técnica e funcional, além de melhorias no desempenho operacional, que possam permitir e indicar a sua aplicação em processos de escala industrial. A partir dos resultados obtidos neste trabalho, foi possível constatar que esta plataforma de controle automático denominada Arduino é muito atrativa em termos econômicos, de fácil implementação e precisa nas ações de controle de nível em escala piloto, indicando ser uma plataforma com grande potencial e viabilidade nas aplicações em processos de escala industrial.
\end{abstract}

\section{INTRODUÇÃO}

Mundialmente, a automação dos processos industriais se torna cada vez mais comum em indústrias de grande, médio e até de pequeno porte. Isso ocorre, em função do enorme benefício que os controles representam nos processos, por diminuir oscilações de resultados, aumentar a eficiência na conversão de reações químicas e das linhas de processamento pela sua precisão. Também, são aplicados controles em processos visando tanto a segurança quanto a melhoria dos sistemas de alívio de pressão em linhas, de controle de temperatura em reações químicas, bem como no controle de emissão de poluentes para atender as legislações ambientais vigentes.

Neste artigo é apresentado o processo de implantação de um método automático de controle de nível em um tanque com alimentação constante e controlada, com o intuito de se estudar o seu potencial para a aplicação industrial. Esse método de controle se baseia na 
utilização de um microcontrolador denominado Arduino, devidamente programado para controlar a alimentação e descarga desse tanque, em função do seu nível, por meio de sinais coletados através de dois sensores de nível, instalados internamente na base e no topo do tanque.

A implantação de um método de controle automático via Arduino nesse processo em escala piloto, teve por finalidade verificar possíveis contribuições em termos de viabilidade econômica, técnica e funcional, além de melhorias no desempenho operacional, que possam permitir e indicar a sua aplicação em processos de escala industrial. A partir dos resultados obtidos com este trabalho, será possível vislumbrar a viabilidade das aplicações em que ele possa se encaixar dentro das indústrias. Vale ressaltar, que o processo de controle de nível automático já é um processo bastante utilizado dentro da indústria. Porém, levando-se em conta o grande aumento na utilização do Arduino como objeto de controle para diversas aplicações, bem como o seu baixo custo e grande eficiência nas funções para qual se aplica, este projeto nos permite avaliar se a sua implantação tem a capacidade de retornar uma eficiência satisfatória frente à sistemas de controle mais sofisticados e, consequentemente, até que ponto seria interessante utilizar este método dentro da indústria de grande porte tendo em vista melhorias no processo e diminuição de custos.

\section{O ARDUINO E O SEU DESTAQUE NO CONTROLE DE PROCESSOS}

O Controle Automático tem desempenhado um papel vital no avanço da engenharia e da ciência, tornando-se parte importante e integrante em processos industriais e de manufatura modernos, além da sua extrema importância para os veículos espaciais, sistemas de guiamento de mísseis, sistemas robóticos, e similares (Ogata, 2011).

Bouyer (2008) diz que, controlar um processo contínuo, por intermédio de sistema automatizado, implica em colocar os saberes e a cognição incorporada em funcionamento para manter o processo de produção dentro dos parâmetros estabelecidos e da normalidade. A finalidade de se projetar um sistema de controle que consiste de componentes interconectados é a de se realizar um objetivo desejado com uma maior precisão e, para a compreensão desta finalidade, é útil recorrer aos exemplos de controles ao longo da história, pois estes sistemas incorporam grande parte das ideias de retroação usadas atualmente (Dorf e Bishop, 2013).

Hoje em dia, vê-se constantemente inovações quanto aos sistemas automáticos em diversos segmentos do mercado como na telefonia, informática, sistemas inteligentes de navegação veicular, sistemas de automação em residências e, é claro, grandes investimentos em sistemas de controle automático para processos industriais.

Uma das inovações mencionadas é, sem sombra de dúvidas, a Plataforma Arduino. Esta placa vem ganhando cada vez mais espaço no cenário dos sistemas de controle automático e, na maioria dos casos, se mostra muito eficiente e economicamente viável. Teve seu lançamento no ano de 2005 e, desde então, mais de 150.000 placas Arduino foram vendidas em todo o mundo. Este número, porém, não leva em consideração as placas-clone desenvolvidas com base neste dispositivo, o que pode fazer com que o número de vendas, suba para mais ou menos 500.000 placas (McRoberts, 2011). Tais números contribuíram para 
que, em 2006, o projeto Arduino recebe-se uma menção honrosa na categoria Comunidades Digitais, que foi promovido pela Prix ArsEletronics (Abreu, 2012).

Tanto sucesso é atribuído à enorme facilidade de manipulação da placa, tornando possível que pessoas que não tenham conhecimento algum de programação e eletrônica, possam fazer seus projetos em aplicações de qualquer natureza, além do fato de que tanto o hardware, quando o software, são open-source, permitindo a sua aplicação e modificação por infinitas vezes. A variedade de modelos que esta placa possui, permite a sua aplicação a uma infinidade de situações e, para facilitar ainda mais o seu uso, muitas destas aplicações são disponibilizadas pela comunidade que o utiliza. Basta realizar uma pesquisa na internet com a palavra "Arduino" e milhares de resultados estarão disponíveis em fração de segundos, com explicações e exemplos de circuitos e códigos prontos, desenvolvidos por diversos usuários da plataforma, dos quais podem ser feitas modificações particulares para utilização em projetos pessoais (McRoberts, 2011). O Arduino ainda possui a sua própria linguagem de programação, a qual é mencionada em Souza et al. (2011) como Linguagem Processing, tendo como base a Linguagem $\mathrm{C} / \mathrm{C}++$, preservando sua sintaxe e suas características de linguagem. As modificações apenas tornaram a programação mais prática e intuitiva, de fácil desenvolvimento, para que o Arduino se tornasse ainda mais chamativo à aqueles que possuem pouco, ou nenhum conhecimento em programação. Tudo isso pode ser desenvolvido no software IDE Arduino (IDE - Internal Development Environment - Ambiente Interno de Desenvolvimento), que possui uma interface intuitiva e de fácil interpretação.

Dentre os modelos de placas disponíveis está o Arduino Uno, que já possui sua $3^{\mathrm{a}}$ revisão, sendo o modelo mais atual disponível no mercado e que foi o escolhido para servir de base para este projeto. Ele é um modelo bem completo e possui todas as características necessárias para atender à maioria dos projetos de aplicação possíveis para a plataforma. "Uno", significa "Um" em italiano, e foi atribuído a este modelo para marcar o lançamento do Arduino 1.0 (Arduino, 2014). O modelo Uno do Arduino pode ser visto na Figura 1, logo abaixo.

Figura 1 - Arduino Uno R3.

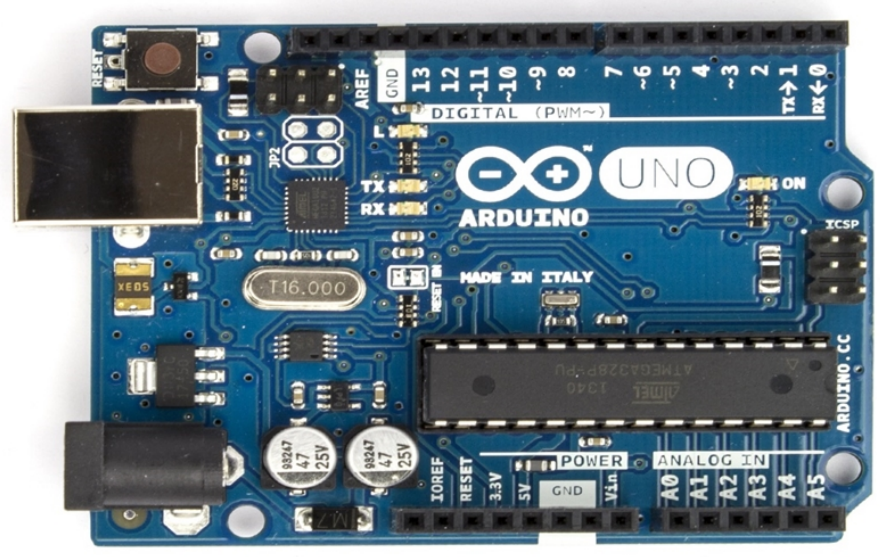




\section{MONTAGEM E FUNCIONAMENTO DO PROJETO}

A montagem do projeto, como já foi mencionado anteriormente, foi realizada em escala piloto nas dependências do Laboratório de Engenharia Química do UNIFAE. Foram inseridos, no Reservatório $\mathrm{A}$, dois sensores de nível (máximo e mínimo). Também neste reservatório, foram afixadas duas minibombas responsáveis por fazer a circulação da água de um reservatório para o outro em um ciclo fechado. No Reservatório B, foi alocada uma Válvula Solenoide Automática, conectada em série à mini bomba de alimentação de água e a um sensor de medição de fluxo. Quando o sensor de nível mínimo do Reservatório A é ativado, a válvula solenoide é aberta ao mesmo tempo em que a mini bomba de alimentação é ativada. Esse processo garante a circulação de água do Reservatório B para o Reservatório A. Adicionalmente, o fluxo de água de alimentação que sai da mini bomba é monitorado pelo sensor de medição de fluxo, com a finalidade de se detectar possíveis flutuações no fluxo de bombeamento.

Do ponto de vista eletrônico, o ciclo do sistema começa quando o Reservatório A atinge o seu nível máximo, que é indicado quando o sensor de nível instalado em seu topo entra na posição $O n$ e, com isso, há o envio de um sinal para o Arduino. Ao receber este sinal, o Arduino faz sua leitura e envia uma "ordem" para que haja o acionamento da mini bomba de esvaziamento. Com isso, a água começa a ser circulada do Reservatório A para o Reservatório B. Esta circulação, entretanto, não pode ser encerrada quando o sensor de nível máximo entre em sua posição Off pois, embora o nível tenha baixado, o reservatório ainda está praticamente cheio. Sendo assim, a circulação só é encerrada quando o sensor de nível mínimo do Reservatório A entra em sua posição On, o que indica que toda a água do reservatório foi drenada. Neste momento, outro sinal é enviado ao Arduino, desta vez, pelo sensor de nível mínimo. Este sinal é interpretado e outra "ordem" é enviada pelo Arduino para que a mini bomba de esvaziamento seja desligada ao mesmo tempo que a mini bomba de alimentação e a solenoide do Reservatório B sejam acionadas, possibilitando o processo inverso do explicado anteriormente. A partir desse comando, o Reservatório A começa a ser alimentado novamente e a mini bomba de alimentação, assim como no caso da mini bomba de esvaziamento, só poderá ser desligada quando o sensor de nível máximo entrar em sua posição $O n$, indicando que o reservatório está completamente cheio. Com isso tem-se um ciclo completo, que se repete continuamente em função da alternância de acionamento entre os sensores de máximo e mínimo instalados no Reservatório A, os quais enviam sinais para o Arduino, que procederá com as ações de controle on/off programadas na IDE, para assegurar o esvaziamento e a alimentação do Reservatório A. Os LED's instalados no projeto indicam a posição dos sensores, mostrando se os mesmos estão no estado $O n$ ou no estado Off. Isso ajuda a monitorar cada etapa do ciclo de funcionamento do sistema. Quando os dois sensores estão na posição On, os LED's verdes se acendem para indicar tal estado. Conforme o nível do reservatório vai abaixando, os sensores voltam para a posição Off e, consequentemente, os LED's verdes se apagam e, ao mesmo tempo, os LED's vermelhos se acendem, indicando o estado dos sensores.

Em função desta alternância de ações comandadas pelos sinais enviados através dos sensores, fez-se necessário desenvolver um código fonte específico para o projeto citado. Desta forma, pode-se programar exatamente as ações para a finalidade que foi elaborada 


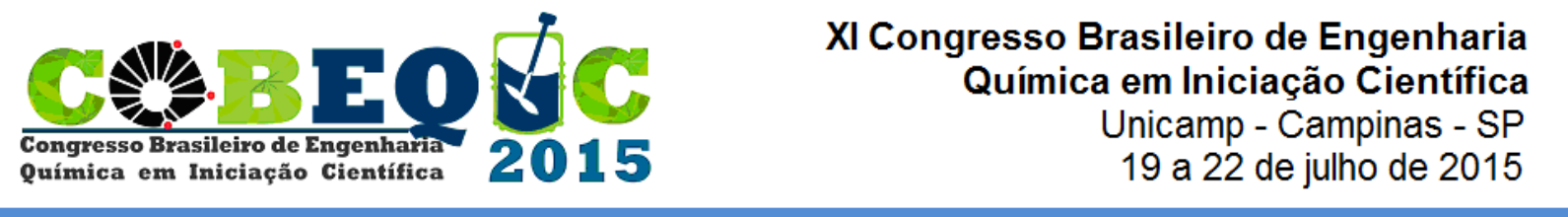

desde o princípio do desenvolvimento deste projeto. O projeto final pode ser visto na Figura $2, \log$ a abaixo.

Figura 2 - Projeto Final do Sistema de Controle de Nível

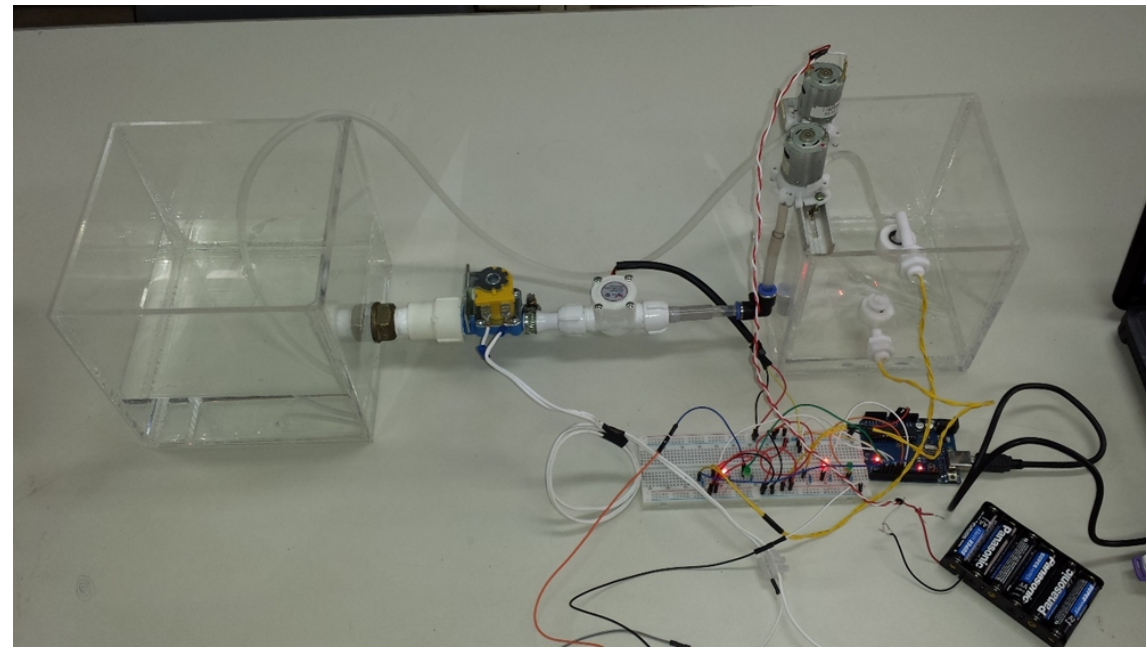

\section{CONCLUSÃO}

Os resultados obtidos com o projeto final foram satisfatórios, mostrando que o Arduino possui um grande potencial de aplicação para sistemas de controle de nível em diversas áreas, desde residências, para se controlar o nível da caixa d'água até a grandes indústrias, para se controlar a alimentação de um tanque em uma estação de tratamento de água, por exemplo.

Quanto à aplicação deste sistema em escala industrial, algumas considerações devem ser feitas como, por exemplo, o fato de ser necessário embarca-lo para que se torne viável tal aplicação. Embarcar o Arduino nada mais é do que acoplar a placa em uma caixa e afixá-la em um local específico que seja destinada à área de controle e automação da indústria e, também, fixar todos os fios em suas respectivas portas no Arduino, salvo o fato de que na indústria não se tem a opção de deixar estes fios conectados de maneira móvel na placa.

O desenvolvimento do código-fonte através da IDE do Arduino se mostrou relativamente simples em comparação com outras linguagens de programação de alto nível, apesar da complexidade no desenvolvimento do código para a finalidade específica do projeto, sendo assim uma alternativa bastante favorável para quem ainda não tem tanto conhecimento em programação.

Os propósitos do desenvolvimento deste projeto, que eram de controlar a alimentação e a descarga de água em um reservatório garantindo que o mesmo não transbordasse ao ser alimentado e que, tampouco, sua mini bomba de esvaziamento tivesse seu funcionamento comprometido por operar com o reservatório em nível insuficiente, também foram cumpridos de forma satisfatória. 
Por fim, como mencionado anteriormente, os custos se mostraram relativamente baixos para sua aplicação, o que tornou a sua utilização para este tipo de aplicação ainda mais atrativa. Na Tabela 1, são mostrados os custos relacionados à montagem do projeto.

Tabela 1 - Custo Médio do Projeto de Controlador de Nível

\begin{tabular}{l|c|c|c}
\hline Dispositivo & Custo Médio & $\begin{array}{c}\text { Quantidade Utilizada no } \\
\text { Projeto }\end{array}$ & Custo Final \\
\hline Kit Arduino Uno & $\mathrm{R} \$ 150,00$ & 1 & $\mathrm{R} \$ 150,00$ \\
\hline Sensor de Nível & $\mathrm{R} \$ 14,00$ & 2 & $\mathrm{R} \$ 28,00$ \\
\hline Válvula Solenoide & $\mathrm{R} \$ 13,50$ & 1 & $\mathrm{R} \$ 13,50$ \\
\hline Mini Bomba & $\mathrm{R} \$ 30,00$ & 2 & $\mathrm{R} \$ 60,00$ \\
\hline Sensor de Fluxo & $\mathrm{R} \$ 40,00$ & 1 & $\mathrm{R} \$ 40,00$ \\
Hall & & & $\mathrm{R} \$ \mathbf{2 9 1 , 5 0}$ \\
\hline
\end{tabular}

\section{REFERENCIAS}

ABREU, A D S. Arduino - Plataforma Eletrônica Microcontrolada. 2012. 124p. Dissertação (Bacharel) - Centro de Ciências Exatas e Tecnologia - Departamento de Engenharia de Eletricidade, Universidade Federal do Maranhão, São Luís, 2012.

ARDUINO. Disponível em: http://www.arduino.cc. Acesso em: 25/03/2015

BOUYER, G C. A Mente Incorporada no Controle de Processo Contínuo: ação, cognição e comunicação na atividade de trabalho. In Gestão \& Produção, v. 15, n.3. p. 539-550, 2008 .

DORF, R C e BISHOP, R H. Sistemas de Controle Moderno. 12 ed. Rio de Janeiro: LTC, 2013.

MCROBERTS, M. Arduino Básico. São Paulo: Novatec, 2011.

OGATA, K. Engenharia de Controle Moderno. 5 ed. São Paulo: Pearson, 2011.

SOUZA, A R; PAIXÃO, A C; UZÊDA, D D; DIAS, M A; DUARTE, S; AMORIM, H S. A Placa Arduino: uma opção de baixo curto para experiências de física assistidas pelo PC. In Revista Brasileira de Ensino de Física, v.33, n.1. 1702. Instituto de Física, Universidade Federal do Rio de Janeiro. Rio de Janeiro, 2011. 